JIA: 1st level: etanercept; 2nd: adalimumab or abatacept. Data collected: age, sex, diagnosis, drug used after protocol implementation, previous treatments and reasons for changing. Data Source: medical records, pharmacy database. Statistical analysis: SPSS 15.0.

Results Of 455 patients receiving BT, 73 met the inclusion criteria: $53.4 \%$ were beginning their treatment, $46.6 \%$ were treatment changes. Median age: 51.1 ( \pm 11.5$)$ years, $76.7 \%$ were women. The percentages by pathology and gender were: RA 56.9\% (women: 95.1\%), SAPs $43.1 \%$ (women: $51.6 \%)(p<0.05)$. Patients starting treatment: RA: $52.7 \%$, SAPs $47.3 \%$. Administration routes and drugs used: RA: subcutaneous (95.0\%) and etanercept $(90.0 \%)$; SAPs: subcutaneous (94.5\%) and etanercept (66.7\%). Changes in treatment: RA $61.8 \%$, SAPs $38.2 \%$, drug used after switching: RA (adalimumab 33.3\%, etanercept $28.6 \%$, tocilizumab $14.3 \%$, rituximab 9.5\%), SAPs (golimumab 61.5\%, adalimumab 30.8\%, etanercept $7.7 \%)$. The reasons for switching were ineffectiveness $(91.2 \%)$ and intolerance (8.8\%).

Conclusions The overall level of compliance with the protocol was high. The most widely prescribed drug in treatment-naive patients was etanercept. Adalimumab was the most prescribed in patients who switched treatment. Lack of effectiveness was the main reason for changing treatment.

No conflict of interest.

\section{OHP-049 IMPROVING COMPLIANCE AID DISPENSING FOR PATIENTS DISCHARGED FROM NORTH BRISTOL NHS TRUST (NBT) BY USING A 3RD PARTY DISPENSING PARTNERSHIP}

doi:10.1136/ejhpharm-2013-000276.423

J Smith, CE Church. North Bristol NHS Trust, Pharmacy, Bristol, UK

Background North Bristol NHS Trust (NBT) is a large teaching hospital split over two main sites. Despite NBT Pharmacy implementing an assessment tool to minimise inappropriate use of compliance aids, demand still doubled in 5 years to over 200 a month exceeding capacity and causing:

- Delayed discharges

- Inappropriate discharges

- Non-compliance

- Re-admissions

- Complaints

Purpose To improve the provision of compliance aids in accordance with the objectives of the Quality, Innovation, Productivity and Prevention (QIPP) programme by:

- Managing the increasing workload

- Reducing discharge waiting times

- Reducing length of stay

- Supporting patients managing medicines at home

Materials and Methods A phased and innovative solution of outsourcing work to a commercial community partner without significant upfront funding was devised. Initially a 'Small Tests of Change' pilot project was launched with a community pharmacy (Lloydspharmacy) to trial the provision of a dispensing service against a service level agreement. After proving the concept and refining the process this was expanded into an independently managed central facility (provided by Lloydspharmacy and the wholesaler AAH Pharmaceuticals) known as the 'The Hub' which serves NBT's multiple sites.

Results This innovative approach has enabled NBT Pharmacy in accordance with OIPP objectives to improve the quality of service and manage a doubling in workload within existing budgetary constraints. The Hub now supports $90 \%$ of NBT's compliance aid dispensing and the average turnaround time has reduced from more than 48 hours to 24 hours (see Table)
Conclusions NBT Pharmacy has developed an innovative and mutually beneficial partnership between an acute NHS Trust and a commercial service provider resulting in the successful management of increasing demand for compliance aids on discharge.

\section{Abstract OHP-049 Table}

\begin{tabular}{|c|c|c|c|c|}
\hline Phase & Description & $\begin{array}{l}\text { Turnaround } \\
\text { SLA (Hours) }\end{array}$ & $\begin{array}{l}\text { Monthly } \\
\text { Rate (Peak) }\end{array}$ & Duration \\
\hline Pre-Pilot & Performed in house. & $N / A>48$ & 40 & Pre - Feb 09 \\
\hline Phase 1 & $\begin{array}{l}\text { Pilot with Lloydspharmacy } \\
\text { Community Branch. }\end{array}$ & $22-46$ & 74 & 18 Months \\
\hline Phase 2 & $\begin{array}{l}\text { Pilot with managed central service } \\
\text { provide by The Hub. }\end{array}$ & $21-45$ & 61 & 2 Months \\
\hline Phase 3 & Service extended to both NBT sites. & $21-45$ & 105 & 5 Months \\
\hline Phase 4 & $\begin{array}{l}\text { Service levels increased to reduce } \\
\text { maximum turnaround time. }\end{array}$ & $21-26$ & 206 & Post Feb 11 \\
\hline
\end{tabular}

No conflict of interest.

\section{OHP-050 IN VITRO EVALUATION OF A NEW SAFETY CONNEXION FOR THE ADMINISTRATION OF ENTERAL NUTRITION: ENLOCK}

doi:10.1136/ejhpharm-2013-000276.424

'B Dekyndt, ${ }^{1} \mathrm{~F}$ Bourdon, 'L Castelain, 'S Neuville, ${ }^{2 B}$ Décaudin, ${ }^{3 P}$ Odou. ${ }^{\top} \mathrm{CHRU} D E$ LILLE, Hospital Pharmacy, Lille, France, ${ }^{2}$ University Lille Nord de France, Pharmacy, Lille, France; ' ${ }^{U}$ DSL EA GRIIOT, UFR Pharmacie, Lille, France

Background New ENLock standard connectors (between the administration tube/enteral feeding catheter-EFC) and ENPlus (between nutrition bag/administration tube) are designed to administer enteral nutrition (EN) safely and avoid unsafe connexions. These connectors will coexist for a while with devices not equipped with them. Compatibility data will be necessary to ensure they are used safely.

Purpose In vitro tests to evaluate the compatibility between devices with and without these connectors.

Materials and Methods The evaluation focused on 12 EFC references from 6 suppliers, 4 nutrition bags from 4 suppliers and one administration tube with an ENLock connexion at both extremities (Nutricia). Following the NF-EN-1615 and 1618 norms, five tests were performed in triplicate on each reference. (1) A leak test required eosin solution and simulated pressure of $50 \mathrm{kPa}$ for $2 \mathrm{~min}$ in the connectors. Leaks were revealed by spots on philtre paper. (2) An EFC connector deformability test was performed by measuring the inner and outer diameter with callipers after one connexion/ disconnection a day for 30 days. (3-4) Two penetration tests, ENLock/EFC and ENPlus/bag, were performed using a dynamometer, exerting a linear force and measuring the maximum force for connexion. (5) An EFC connexion resistance test was performed using a dynamometer exerting a linear traction and measuring the maximum force for disconnection.

Results No leaks were detected. The changes in the EFC connector internal and external diameters tested were respectively up to $30 \%$ and $6 \%$. The tube connexion force to the EFC ranged from $37.8 \mathrm{~N}$ to $216.2 \mathrm{~N}$ and to the bag from $14.6 \mathrm{~N}$ to $20.1 \mathrm{~N}$. Two suppliers' bags could not be connected to the ENPlus device. The tube disconnection force to the EFC ranged from $3.2 \mathrm{~N}$ to $44.8 \mathrm{~N}$ (limit value: $15 \mathrm{~N})$.

Conclusions Important differences exist between suppliers. Some feeding lines don't meet the specific EN norms for these devices particularly as regards the maximum disconnection force so their use with incompatible devices can be risky.

No conflict of interest. 